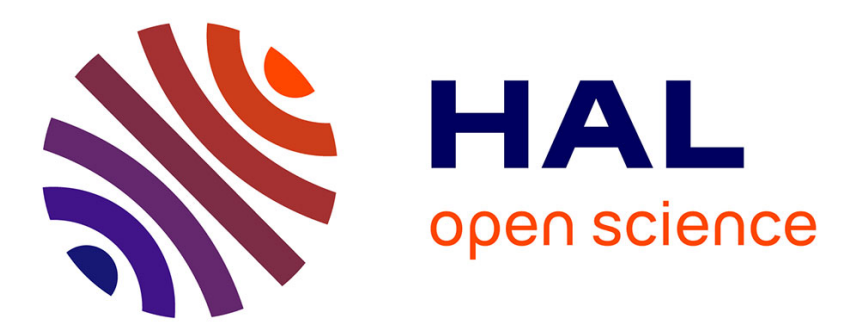

\title{
Investigation of the mobile granular layer in bedload transport by laminar shearing flows
}

Pascale Aussillous, Julien Chauchat, Mickael Pailha, Marc Médale, Elisabeth Guazzelli

\section{- To cite this version:}

Pascale Aussillous, Julien Chauchat, Mickael Pailha, Marc Médale, Elisabeth Guazzelli. Investigation of the mobile granular layer in bedload transport by laminar shearing flows. Journal of Fluid Mechanics, 2013, 736, pp 594-615. 10.1017/jfm.2013.546 . hal-00915257

\section{HAL Id: hal-00915257 https://hal.science/hal-00915257}

Submitted on 6 Dec 2013

HAL is a multi-disciplinary open access archive for the deposit and dissemination of scientific research documents, whether they are published or not. The documents may come from teaching and research institutions in France or abroad, or from public or private research centers.
L'archive ouverte pluridisciplinaire HAL, est destinée au dépôt et à la diffusion de documents scientifiques de niveau recherche, publiés ou non, émanant des établissements d'enseignement et de recherche français ou étrangers, des laboratoires publics ou privés. 


\title{
The erosion of granular beds under the action of laminar shearing flows
}

\author{
PASCALE A USSILLOUS ${ }^{1}$, JULIEN CHAUCHA T ${ }^{2}$, \\ MICKAEL PAILHA ${ }^{3}$, A RC MÉDALE \\ AND ÉLISABETH G U A ZZELLI \\ ${ }^{1}$ Aix-Marseille Université, CNRS, IUSTI UMR 7343, 13453 Marseille Cedex 13, France \\ ${ }^{2}$ LEGI, UJF/INPG/CNRS, BP 53, 38041 Grenoble, France \\ ${ }^{3}$ LOCIE, CNRS - Université de Savoie, 73376 Le Bourget du Lac Cedex, France
}

(Received 26 June 2013)

The mobile layer of a granular bed composed of spherical particles is experimentally investigated in a laminar rectangular-channel flow. Both particle and fluid velocity profiles are obtained using particle image velocimetry for different index-matched combinations of particles and fluid and for a wide range of fluid flow-rates above incipient motion. A full three-dimensional investigation of the flow field inside the mobile layer is also provided. These experimental observations are compared to the predictions of a two-phase continuum model having a frictional rheology to describe particle-particle interactions. Different rheological constitutive laws having increasing degree of sophistication are tested and discussed.

\section{Introduction}

The erosion of granular beds under the action of fluid shearing flows is a problem which has been continuously studied and discussed for over a century. This phenomenon is indeed encountered in a wide range of processes of important relevance in nature or industry such as sediment transport in rivers or oceans and slurry transport in the mining and petroleum industry. The interaction of the flow with the erodible bed can give rise to self-formed morphologies such as ripples and dunes. While, in most of the natural occurrence of these patterns, the flow is likely turbulent, it is not necessary and laminar flows also generate such features. The similarity in observed morphologies seem to reflect analogies in the underlying physics and thus using laminar flow can be a simplest way in the laboratory to obtain insights into many aspects of sediment transport and morphodynamics (Lajeunesse et al. 2010). The present paper focusses on particle transport in the laminar regime and in particular on the mode of particle motion in which particles roll and slide but stay in continuous contact within the bed, a situation usually referred to as 'bedload' transport (see e.g. Van Rijn 1984).

There are two basic avenues to tackle the modelling of sediment transport by viscous shearing flow, one being erosion-deposition models and the second being continuum modelling. The erosion-deposition model is based on mass conservation of the particles in a mobile monolayer and requires knowledge of the deposition and erosion rates (see e.g. Charru et al. 2004). Continuum modelling uses mass conservation and momentum balance and the influence of the particles is captured through rheological laws that can be developed from independent experiments. One of the first attempt at this latter type of modelling is due to Bagnold (1956) who used a viscous rheology for the granular stress deduced from rheological measurements of suspensions of neutrally buoyant particles sheared in an annular Couette flow (Bagnold 1954). This continuum approach has 
been recently rationalised by Ouriemi et al. (2009) who developed a two-phase model having a Newtonian rheology for the fluid phase and frictional rheology for the particle phase. While the erosion-deposition model is likely to be appropriate close to incipient particle-motion where only one particle layer is mobile, the continuum approach is more realistic at some distance from onset of motion as the mobile layer can become much larger than one particle diameter.

Two-phase modelling offers a general framework to study the flow of dense granular material. The challenge is however to find the constitutive laws which account for the rheological behaviour of the mobile particulate system. Ouriemi et al. (2009) adopted a simple Coulomb friction for the rheology of the particle phase and showed that this roughly accounted for the observed cubic variation of the particle flux with the bed shear-stress in sediment-transport experiments in laminar pipe flow. However the particle flux was inferred indirectly through bed-height-evolution measurements and not from direct measurement of particle motion inside the moving sediment. To our knowledge only two studies have attempted to perform such investigation of the mobile granular layer. Lobkovsky et al. (2008) examines individual particle motion using matched-index technique in a rectangular pipe in laminar-flow conditions. These experiments provide flow observation inside the mobile bed but only one velocity profile and one concentration profile were shown with a spatial resolution too poor to provide meaningful comparison with any predictions (4 data points in their figure 2). Mouilleron et al. (2009) used the same matched-index technique to measure the velocity profiles of the fluid and particles inside the mobile granular layer in a viscous Couette flow. Only six profiles were obtained with a better but still low spatial resolution ( 5 to 10 data points in their figure 4 ). It should also be stressed that, in this Couette geometry, the thickness of the mobile layer is at most two particle diameters and thus the validity of the continuum approach can be questioned. In these two studies, the particle and fluid properties were not varied which precludes any testing of the scaling law for sediment transport. There is thus a need for accurate measurements inside the mobile granular layer which yield precise comparison with the prediction of the two-phase modelling and testing of the rheological constitutive laws. This is precisely the objective of the present work.

Following previous experiments (Goharzadeh et al. 2005; Lobkovsky et al. 2008), we use a matched-index technique to investigate the mobile layer of a granular bed composed of spherical particles in a rectangular channel flow (see $\S 2)$. The originality and relevance of the experiments lie in the use of different combinations of particles and fluid as well as in the joint measurements of the velocity profiles of the particles and the fluid with unprecedented spatial and temporal resolutions for a wide range of fluid flow-rates above incipient motion (typically 300 profiles comprising 4 to 50 data points). The choice of a Poiseuille over a Couette flow is motivated by the fact that the pressure gradient which drives the flow can produce a large particle flux involving a mobile layer much thicker than one particle diameter (up to 25 particle diameters in the present experiment). This justifies the comparison with a continuum modelling. The experimental observations are thus compared in $\S 4$ to the predictions of the two-phase modelling of Ouriemi et al. (2009) and its full three-dimensional numerical implementation that are described in $\S 3$. We test three types of rheological laws of increasing sophistication: (i) the Coulomb model with constant friction coefficient previously used by Ouriemi et al. (2009), (ii) the granular frictional rheology with a shear-rate-dependent friction coefficient which has been proposed for dense dry granular flows (see e.g. Forterre \& Pouliquen 2008) and extended to particles suspended in a fluid (Cassar et al. 2005), and (iii) a recent rheological model proposed by Boyer et al. (2011) which derives from pressure-imposed rheological measurements of dense suspensions of neutrally-buoyant spheres and unifies 
(a)

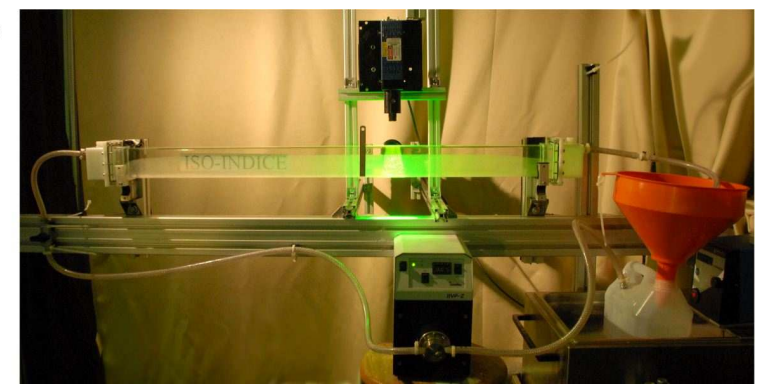

(b)

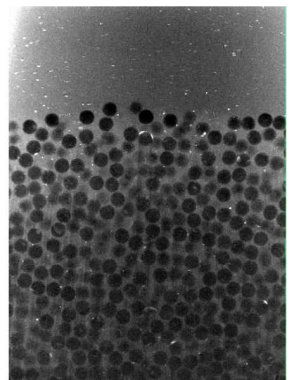

Figure 1. (a) Experimental apparatus. (b) Typical image of the bed test section for run 15 (see corresponding movie 1).

\begin{tabular}{|c|c|c|c|c|c|c|c|}
\hline Combination & Particles & Fluids & $\begin{array}{c}d \\
(\mathrm{~mm})\end{array}$ & $\begin{array}{c}\rho_{p} \\
\left.\text { (g.cm } \mathrm{cm}^{-3}\right)\end{array}$ & $\begin{array}{c}\rho_{f} \\
\left.\text { (g.cm }{ }^{-3}\right)\end{array}$ & $\begin{array}{c}\eta \\
(\mathrm{cP})\end{array}$ & $\mathrm{n}$ \\
\hline$A$ & Borosilicate & $\begin{array}{l}\text { Water }(15 \% \text { wt }) \\
+ \text { Triton X-100 }\end{array}$ & $1.1 \pm 0.1$ & 2.23 & 1.06 & 320 & 1.47 \\
\hline$B$ & PMMA & Triton X-100 & $2.04 \pm 0.03$ & 1.19 & 1.07 & 270 & 1.49 \\
\hline
\end{tabular}

the rheological behaviour of dense suspensions and granular media. We also provide a full three-dimensional investigation of the flow field inside the mobile layer. Conclusions regarding the validity of the different rheological models are drawn in $\S 5$.

\section{Experiments}

We use a match-index technique to investigate the mobile granular layer for the sediment transport of spherical particles in a rectangular channel in the viscous laminar regime. The apparatus consists of a horizontal glass tube (of height $H=6.5 \mathrm{~cm}$, width $W=3.5 \mathrm{~cm}$, and length $100 \mathrm{~cm}$ ) partially filled with particles, as shown in figure 1(a). Two index-matched combinations of particles and fluid are used, see table 1 . At the outlet from the channel, the fluid is run into a thermostated fluid reservoir, which insures a constant temperature of $25^{\circ} \mathrm{C}$ across the whole flow loop. From this reservoir, it is returned by a gear pump (Ismatec) into the channel. At the inlet, the fluid goes through a packed bed of large spheres providing a homogeneous and laminar flow. The pump produces a pulseless fluid delivery and consequently a constant fluid flow-rate $Q_{f}$ which is determined by measuring a collected volume of fluid in a given time.

A given amount of particles is introduced inside the tube which is then filled by the fluid. The granular bed is then prepared using the following steps. The tube is (i) flipped down, (ii) tilted at an angle of $43^{\circ}$ with the fluid input end at the bottom, and (iii) set back up horizontally. The duration of each step is set at the same sedimentation time (2 and 4 minutes for combinations $A$ and $B$, respectively). This procedure fills the tube top leaving an empty buffer space near the outlet. (iv) A small flow rate is applied during typically 4 minutes for creating a small uniform gap (typically 5 grain diameters) devoid of particles at the top. These fourth steps are repeated to create a reproducible initial condition for all runs. After the preparation phase, a given flow rate is imposed and kept constant for the duration of each run (typically $3-5$ minutes). Eroded grains fall 


\begin{tabular}{|c|c|c|c|c|c|c|c|c|c|c|c|}
\hline Run & Combi. & $Q\left(\mathrm{~m}^{3} / \mathrm{s}\right)$ & sym. & Run & Combi. & $Q\left(\mathrm{~m}^{3} / \mathrm{s}\right)$ & sym. & Run & Combi. & $Q\left(\mathrm{~m}^{3} / \mathrm{s}\right)$ & sym. \\
\hline 1 & A & $2.710^{-6}$ & $\times$ & 7 & A & $6.910^{-6}$ & $\bullet$ & 13 & B & $3.210^{-6}$ & $\Delta$ \\
\hline 2 & $A$ & $3.610^{-6}$ & $\circ$ & 8 & $A$ & $8.210^{-6}$ & $\triangle$ & 14 & $B$ & $3.610^{-6}$ & $\square$ \\
\hline 3 & $A$ & $4.410^{-6}$ & $\Delta$ & 9 & $A$ & $8.610^{-6}$ & $\square$ & $15^{(*)}$ & $B$ & $4.110^{-6}$ & 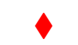 \\
\hline 4 & $A$ & $5.310^{-6}$ & $\square$ & 10 & A & $9.710^{-6}$ & $\vec{\diamond}$ & 16 & $B$ & $4.610^{-6}$ & $\nabla$ \\
\hline $5^{(*)}$ & $A$ & $5.710^{-6}$ & $\diamond$ & 11 & $B$ & $2.210^{-6}$ & $x$ & 17 & $B$ & $5.610^{-6}$ & - \\
\hline 6 & A & $6.110^{-6}$ & $\nabla$ & 12 & $B$ & $2.710^{-6}$ & $\circ$ & & & & \\
\hline
\end{tabular}

TABle 2. Performed runs: the laser sheet is located at the middle of the tube $(17.5 \mathrm{~mm}$ from the front wall) except for runs 5 and 15 [noted by ${ }^{(*)}$ where 7 runs where performed at different locations $(\times) 2.5 \mathrm{~mm},(\circ) 5 \mathrm{~mm},(\boldsymbol{\Delta}) 7.5 \mathrm{~mm},(\square) 10 \mathrm{~mm},(\diamond) 12.5 \mathrm{~mm},(\nabla) 15$ $\mathrm{mm}$, and $(\bullet) 17.5 \mathrm{~mm}$ from the front wall.
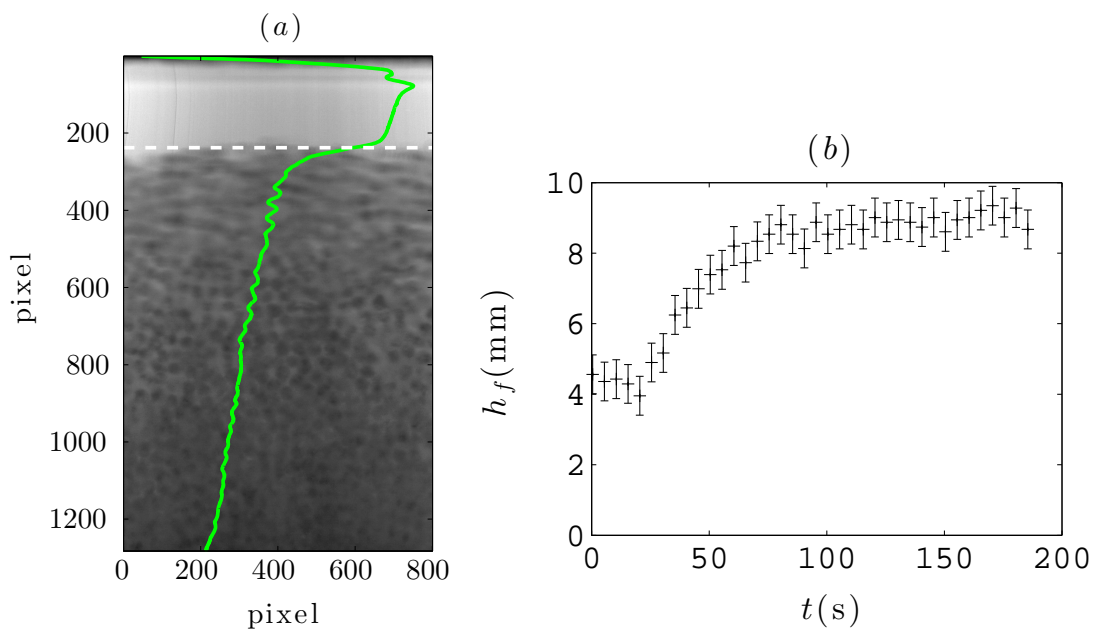

FIGURE 2. (a) Averaged image after $35.5 \mathrm{~s}$ for run 2 with the corresponding averaged grey-level profile ( - , green on line) and detected fluid-bed interface (--, white on line). (b)Temporal evolution of the fluid height $h_{f}$ for the same run.

out into the empty buffer space at the outlet leaving an upstream region exhibiting a flat fluid-particle interface which decreases with time. The characteristics of the different performed runs are summarized in table 2 .

The test section of $4 \mathrm{~cm}$ is located at a distance $\approx 50 \mathrm{~cm}$ from the entrance of the tube. It is illuminated by a green laser sheet (Laser 2000, $532 \mathrm{~nm}, 100 \mathrm{~mW}$ ) aligned with the tube length in its middle for most of the experiments, see figure 1(a). Successive scans are also performed to obtain a three-dimensional investigation of the flow (runs 5 and 15 indicated in table 2). A digital camera (Basler Scout) with a resolution of $1392 \times 1040$ pixels records a sequence of images at a rate of 20 frames per second. A dye (Rhodamine $6 \mathrm{G}$ ) which fluoresces when illuminated by the laser in a wavelength range larger than $555 \mathrm{~nm}$ is added to the fluid. Owing to a red filter (with wavelength band-width 590$1800 \mathrm{~nm}$ ) placed in front of the camera lens, the particles look dark against a bright fluid as seen in figure $1(\mathrm{~b})$.

The fluid height $h_{f}$ above the granular layer is measured every $5 \mathrm{~s}$ by averaging 10 images (corresponding to averaging over $0.5 \mathrm{~s}$ ) and detecting the interface between the fluid and the granular layer by the change of slope in the averaged grey-level profile of the obtained averaged image [see figure 2(a)]. The error bar is estimated to be half a 
grain diameter. A typical temporal evolution of $h_{f}$ is shown in figure 2(b). After a first transient dilation of the granular layer, the particles are eroded out of the test section which induces an increase of $h_{f}$ [for times $t \gtrsim 30 \mathrm{~s}$ in figure 2(b)]. This process slows down with time and eventually stops at a final fluid height (Ouriemi et al. 2007; Lobkovsky et al. 2008). We do not wait long enough to observe the cessation of particle erosion which is reached after typically one hour. The runs are stopped when the thickness of the mobile layer corresponds to $\approx d$. Assuming that the flow is steady after the transient dilatation, the flow properties can be determined at each given $h_{f}$.

Particles velocities are measured by particle image velocimetry (PIV) using the Matlab PIV software DPIVsoft (Meunier \& Leweke 2003). For a given $h_{f}, 10$ pairs of successive images are processed to find the velocity profile, corresponding to increment from 1 $(0.05 \mathrm{~s})$ to $10(0.5 \mathrm{~s})$ images. In practice, this involves using a square interrogation region having a size $S_{\text {ir }}=64$ pixels $(\approx 2 d)$, with 45 boxes on the horizontal direction and 60 boxes in the vertical direction. The local particle displacement at each node for each pair is measured using a cross-correlation. The vertical velocity profile is build up by taking the median of the velocity for the 45 horizontal boxes for the 10 pairs of images, excluding displacements below 1 pixel and above $S_{\text {ir }} / 3$ pixels (see Meunier \& Leweke 2003). The error is given by one standard error. The spatial resolution of the measurement (given by the height of the interrogation region) is $\approx 2 d$ while the velocity resolution (given by the choice of the image pairs) ranges between 0.06 and $20 \mathrm{~mm} \cdot \mathrm{s}^{-1}$. In the case of combination $B$, the fluid is seeded with fine fingerprint powder, see the white dots in figure 1(b). This means that the PIV measurements provide the fluid velocity in the pure fluid phase and a composite velocity between that of the grains and that of the fluid in the granular layer which is determined by the amount of fingerprint powder in this layer. Since this amount is very small, the PIV measurements mainly give the particle velocity. To determine the full fluid velocity profile, the images are thresholded to capture only the fingerprint powder motion and the same PIV technique is used. However, due to some loss of tracers during the thresholding process, the velocity profile is less fully resolved and presents larger error bars.

The grey level intensity gradient [see figure 2(a)] is mainly due to two effects (Dijksman et al. 2012): (i) a linear broadening of the laser line due to the use of a laser line generator with a finite fan angle and (ii) an absorption due to the presence of the dye leading to an exponential decrease in intensity. Assuming that the second effect stays negligible in our experiment, the particle volume fraction is estimated by using the averaged grey-level profile of the 10 images (averaged on the same box size as in the PIV) and scaling it by the grey-level profile of the immobile initial bed which is assumed to be at a constant particle volume fraction $=0.585$. The particle volume fraction can be estimated for combination A but not for combination B because of a poorer contrast.

From these measurements, we obtain approximately 300 particle concentration and velocity profiles at (approximately 10 to 40) different fluid heighs for the 17 different runs which range over a large extent of flow rates and correspond to two different combinations of particles and fluid (see table 2). Typical particle volume fraction and velocity profiles for the two combinations A and B of particles and fluid are shown in figure 3 . The background image corresponds to averaging the 10 images used in the PIV. Two important observations which are in accordance with the previous findings of Lobkovsky et al. (2008) and Mouilleron et al. (2009) can be made. (i) There is no velocity slip between the particles and the fluid inside the mobile granular layer. (ii) The particle volume fraction is approximately constant inside this layer except at the top interface where it vanishes on a distance $\approx 2 d$. From the velocity and concentration profiles, we can deduce the particle flux $q_{p}$ and the thickness $h_{m}$ of the mobile layer. Since the concentration 
(a)

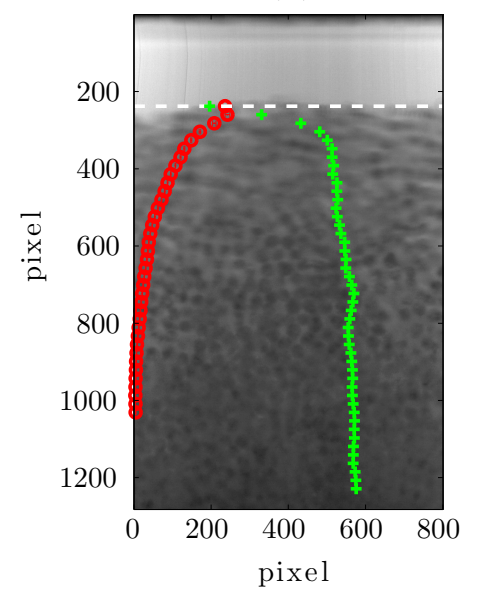

(b)

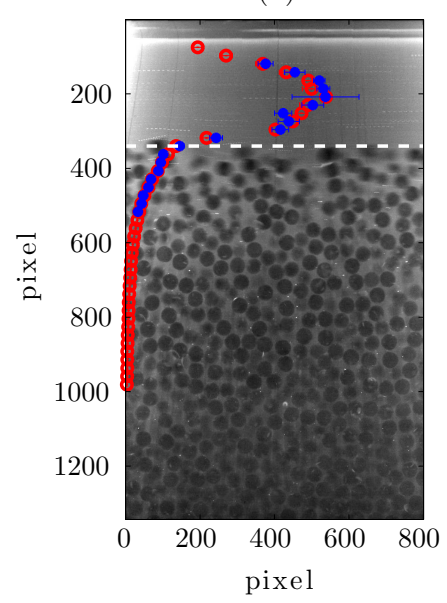

Figure 3. Particle + fingerprint (o, red online) and solely fingerprint ( $*$, blue on line) velocity profiles and particle volume fraction ( + , green online) for (a) run 2 after $35.5 \mathrm{~s}$ (velocity scale $=0.025 \mathrm{~mm} \cdot \mathrm{s}^{-1} \cdot$ pixel $^{-1}$, length scale $=0.029 \mathrm{~mm} \cdot$ pixel $^{-1}$, particle-volume-fraction scale $=0.001$ pixel $^{-1}$, see corresponding movie 2) (b) run 16 after $115.5 \mathrm{~s}$ (velocity scale $=0.025$ $\mathrm{mm} \cdot \mathrm{s}^{-1} \cdot$ pixel $^{-1}$, length scale $=0.046 \mathrm{~mm} \cdot \mathrm{pixel}^{-1}$, see corresponding movie 3$)$. The white horizontal dashed line indicates the fluid-particle interface.

profile is not precisely resolved, it is more convenient to measure the particle-velocity flux $q_{v}$ which is simply given by integrating the particle velocity profile. Note that this $q_{v}$ corresponds to a two-dimensional particle-velocity flux measured in a vertical plane (in the middle vertical plane of the channel for most of the experiments). The thickness $h_{m}$ of the mobile layer is obtained by finding the position inside the layer where a cut-off velocity having a value of $0.09 \mathrm{~mm} . \mathrm{s}^{-1}$ is reached.

We also need to determine the two-dimensional fluid flow-rate $q_{f}$ from the evaluation of the three-dimensional fluid flow-rate $Q_{f}$. The switch to a two-dimensional flow-rate is given by $q_{f}=k Q_{f} / W$ with a geometrical coefficient $k$ which only depends on the aspect ratio $h_{f} / W$ and the lateral position from the center $y / W$

$$
k=\frac{W}{h_{f}} \frac{1-\frac{96}{\pi^{4}} \sum_{n=0}^{\infty} \frac{\cosh \left[(2 n+1) \pi \frac{y}{W}\right]}{(2 n+1)^{4} \cosh \left[\left(n+\frac{1}{2}\right) \pi \frac{W}{h_{f}}\right]}}{\frac{W}{h_{f}}-\frac{192}{\pi^{5}} \sum_{n=0}^{\infty} \frac{\tanh \left[\left(n+\frac{1}{2}\right) \pi \frac{W}{h_{f}}\right]}{(2 n+1)^{5}}} .
$$

The calculation derived from Nicolas et al. (2000) which leads to equation (2.1) assumes a solid bed surface (meaning also no slip boundary condition) and thus expression (2.1) is likely to be valid at relatively small flow rate for which the perturbation induced to the Poiseuille flow by the motion of the granular medium is negligible. In the center of the channel this coefficient $k$ varies from 1.12 to 1.35 in the range of fluid height $h_{f}$ explored in the experiments.

Another important dimensionless number of the problem which controls incipient motion is the Shields number $\theta$. It is basically the shear stress at the top of the bed scaled by the hydrostatic pressure difference across a particle. To infer this number, we follow the same approach as above (Ouriemi et al. 2007). It is given by $\theta=$ $6 k(R e / G a)\left(d / h_{f}\right)^{2}$ where the Reynolds number is $R e=\rho_{f} Q^{f} /(\eta W)$ and the Galileo number $G a=\rho_{f}\left(\rho_{p}-\rho_{f}\right) g d^{3} / \eta^{2}$. 
(a)

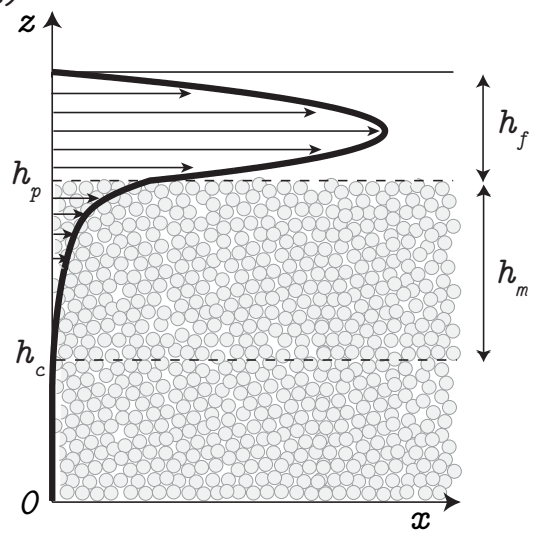

(b)

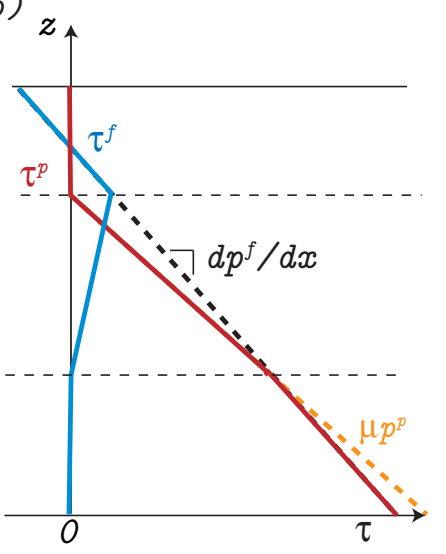

Figure 4. Sketches (a) of the particle bed submitted to a Poiseuille flow and (b) of the exchange between the fluid $\tau^{f}$ (blue online) and particle $\tau^{p}$ (red online) stresses inside the granular bed.

\section{Two-phase modelling}

\subsection{Governing equations}

We use the classical two-phase equations (see e.g. Jackson 1997, 2000) with closures proposed by Ouriemi et al. (2009) as appropriate to the present bed-erosion problem. We consider a flat particle bed of thickness $h_{p}$ submitted to a Poiseuille flow driven by a pressure gradient $\partial p^{f} / \partial x$ in an horizontal channel. For simplicity we focus first on the two-dimensional situation [see figure 4(a)] and the flow is taken as stationary, uniform, and parallel. The velocities thus reduce to their components along the horizontal $x$ direction and are designated as $u^{f}$ for the fluid velocity, $u^{p}$ for the particle velocity, and $U=\phi u^{p}+(1-\phi) u^{f}$ for the volume average velocity, where $\phi$ is the particle volume fraction. The three dimensional implementation of the two phase model is discussed in $\S 3.3$.

The two-phase equations can be written by considering either the two phases (fluid and particles) or one phase (fluid or particles) and the bulk suspension. Considering bulk and fluid phase equations prove to be more convenient as we limit consideration to viscous flow of a granular bed. Along the horizontal $x$-direction, the fluid phase equation reduces to the Brinkman equation (see Brinkman 1947) for the fluid velocity

$$
\frac{\partial p^{f}}{\partial x}-\frac{\partial \tau^{f}}{\partial z}+\frac{\eta}{K}\left(U-u^{p}\right)=0
$$

and the momentum equation for the mixture (particles + fluid) is written as,

$$
\tau^{p}(z)+\tau^{f}(z)=\tau^{f}\left(h_{p}\right)-\frac{\partial p^{f}}{\partial x}\left(h_{p}-z\right)
$$

when integrated from the bed interface position $h_{p}$ to an arbitrary vertical position inside the sediment $z$. The Brinkman equation (3.1) describes in particular the fluid flow through the granular bed. The shear stress of the particle phase is denoted $\tau^{p}$ and the interphase force is the dominant viscous Darcy drag with $\eta$ the fluid viscosity and $K$ the permeability given by the Carman equation $K=(1-\phi)^{3} d^{2} / 180 \phi^{2}$ with $d$ the particle diameter (see e.g. Goharzadeh et al. 2005; Van der Hoef et al. 2005). The mixture equation (3.2) accounts for the transfer between the fluid stress at the top of the bed and the stresses of the fluid $\left(\tau^{f}\right)$ and solid $\left(\tau^{p}\right)$ phases inside the bed. The momentum 
equations along the vertical $z$-direction show that the pressure of the fluid phase along gravity is simply the hydrostatic pressure and that the pressure of the particle phase is proportional to the apparent weight of the solid phase and increases when penetrating inside the bed,

$$
p^{p}=\phi \Delta \rho g\left(h_{p}-z\right),
$$

where $\Delta \rho=\rho_{p}-\rho_{f}$ corresponds to the density difference between the two phases.

\subsection{Closures}

To close equations (3.1) and (3.2), we follow Ouriemi et al. (2009) and consider that the stress tensor of the fluid phase is of Newtonian form $\tau^{f}(z)=\eta_{e}(d U / d z)$ with an effective viscosity $\eta_{e}$ while that of the particle phase comes only from direct particleparticle interactions and is described by a frictional rheology in the mobile granular layer $\left(d u^{p} / d z>0\right)$ for which $\tau^{p}=\mu p^{p}$ with a friction coefficient $\mu$. We have tested three types of constitutive laws of increasing sophistication:

(i) A Coulomb model with constant friction coefficient $\left(\mu=\mu_{s}\right.$, the tangent of the angle of repose) combined with an Einstein effective viscosity $\eta_{e}=\eta(1+5 \phi / 2)$ where the volume fraction is considered constant and $\approx 0.55$ inside the granular layer, i.e. $\eta_{e} / \eta \approx 2.4$.

(ii) A granular frictional rheology which has been proposed for dense dry granular flows (see e.g. Forterre \& Pouliquen 2008) and extended to particles suspended in a fluid (Cassar et al. 2005) with a shear-rate-dependent friction coefficient $\mu(I)=\mu_{1}+I\left(\mu_{2}-\right.$ $\left.\mu_{1}\right) /\left(I+I_{0}\right)$ where $I$ is a dimensionless shear rate which corresponds to the ratio between the time of rearrangement of a particle when it is displaced from its hole and the time taken by the particle to move from one hole to the next $\left(I_{0}, \mu_{1}\right.$, and $\mu_{2}$ are constant which depend upon the particle material and shape used). While for dry granular flows the particulate system is governed by an inertial number $I=d \dot{\gamma}^{p} \sqrt{\rho_{p} / p^{p}}$, it is governed by a viscous number $I_{v}=\eta \dot{\gamma}^{p} / p^{p}$ where $\dot{\gamma}^{p}$ is the particle shear-rate for wet granular flows. For simplicity, the particle volume fraction $\phi$ and the effective viscosity $\eta_{e}$ are again kept constant.

(iii) A suspension rheology with a friction $\mu\left(I_{v}\right)$ with $\mu_{1}=0.32, \mu_{2}=0.7$, and $I_{0}=$ 0.005 which is supplemented by a shear-rate-dependent volume-fraction constitutive law $\phi\left(I_{v}\right)=\phi_{m} /\left(1+I_{v}^{1 / 2}\right)$ and by an effective viscosity $\eta_{e}(\phi)=\eta\left[1+\frac{5}{2} \phi\left(1-\phi / \phi_{m}\right)^{-1}\right]$ which diverges at maximum volume fraction $\phi_{m}=0.585$. This constitutive model has been recently proposed by Boyer et al. (2011) and comes from rheological measurements of dense suspensions of neutrally-buoyant spheres using pressure-imposed rheometry.

\subsection{Implementation}

The two-dimensional calculation can be conducted analytically for the simplest Coulomb model [case (i) of $§ 3.2]$. It is presented with some details here as it gives physical insights of the mechanisms involved as well as the appropriate scalings. There are three key simplifications which permit the calculation to be tackled analytically. First, the friction coefficient, $\mu$, is a constant. Second, the volume fraction $\phi$ is considered constant inside the granular bed and thus the effective viscosity $\eta_{e}$ is also a constant. Third, the Darcy drag term is dominant in the Brinkman equation (3.1). Therefore, inside the bed, there is very little slip between the two phases and both particle and fluid phases move at the velocity of the mixture, $u^{f}=u^{p}=U$. This last assumption is important because the problem is then reduced to simply solving the mixture equation (3.2).

The mixture equation (3.2) shows that the mixture shear stress $\tau^{f}+\tau^{p}$ increases linearly with depth from the surface value $\tau^{f}\left(h_{p}\right)$ due to the horizontal pressure gradient 
$\partial p^{f} / \partial x$. The shear stress of the particle phase is the (constant) friction coefficient, $\mu$, multiplied by the particle pressure given by equation (3.3), i.e. $\tau^{p}=\mu p^{p}=\mu \phi \Delta \rho g\left(h_{p}-z\right)$ while that of the fluid phase is $\tau^{f}=\eta_{e} d U / d z$. Therefore the mixture equation (3.2) can be rewritten as

$$
\mu \phi \Delta \rho g\left(h_{p}-z\right)+\eta_{e} \frac{d U}{d z}=\tau^{f}\left(h_{p}\right)-\frac{\partial p^{f}}{\partial x}\left(h_{p}-z\right),
$$

with zero shear at $z=h_{c}$, zero particle shear stress at $z=h_{p}$, and no slip conditions on the channel boundaries.

Equation (3.4) describes the exchange between the shear stress of the fluid phase $\tau^{f}$ and that of the solid phase $\tau^{p}$ [see figure 4(b)]. On the one hand, the shear stress of the fluid phase $\tau^{f}=\eta_{e} d U / d z=\tau^{f}\left(h_{p}\right)-\left(\mu \phi \Delta \rho g+\partial p^{f} / \partial x\right)\left(h_{p}-z\right)$ is equal to $\tau^{f}\left(h_{p}\right)$ at the top of the granular bed, at $z=h_{p}$, and goes to zero at the bottom of the mobile layer, at $z=h_{c}$ given by $h_{p}-h_{c}=\tau^{f}\left(h_{p}\right) /\left(\mu \phi \Delta \rho g+\partial p^{f} / \partial x\right)$. On the other hand, the shear stress of the particle phase is zero at $z=h_{p}$ and increases inside the bed since $\tau^{p}=\mu p^{p}=\mu \phi \Delta \rho g\left(h_{p}-z\right)$. The particle shear stress can keep the value $\mu p^{p}$ until it reaches $z=h_{c}$ for which granular motion stops.

Equation (3.4) can be solved analytically for this simple Coulomb model to infer the thickness of the mobile granular layer $h_{m}=h_{p}-h_{c}$ and the velocity profile. The thickness of the mobile granular layer is given by

$$
\frac{h_{m}}{h_{f}}=\frac{\eta_{e}}{\eta}\left[\sqrt{1-\frac{\eta}{\eta_{e}} \frac{\partial p^{f} / \partial x}{\partial p^{f} / \partial x+\mu \phi \Delta \rho g}}-1\right],
$$

where $h_{f}$ is the fluid height. This result provides immediate clues about the relevant scalings of the problem. The length scale is given by the fluid height $h_{f}$, the pressure scale by the hydrostatic pressure $\Delta \rho g h_{f}$, and thus the time scale by $\eta / \Delta \rho g h_{f}$ (as suggested by the definition of the viscous number $I_{v}=\eta \gamma^{p} / p^{p}$ ). This relation (3.5) was previously found by Ouriemi et al. (2009) (see their table 4) but in this work the length was made dimensionless by the height of the channel and not by that of the fluid. It should be stressed that these scalings are not restricted to the present Poiseuille problem but also apply for example to a particle bed submitted to a Couette flow, see table 8 of Ouriemi et al. (2009).

Inside the bed $\left(z \leqslant h_{p}\right)$, the velocity profile is

$$
u^{p}=u^{f}=U=\frac{\partial p^{f} / \partial x+\mu \phi \Delta \rho g}{\eta_{e}} \frac{\left(z-h_{c}\right)^{2}}{2},
$$

and is parabolic. It can also be calculated in the pure fluid region $\left(h_{p} \leqslant z \leqslant h_{p}+h_{f}\right)$ and reads,

$$
u^{f}=U=\frac{1}{\eta} \frac{\partial p^{f}}{\partial x} \frac{\left(z-h_{p}-h_{f}\right)\left(z-h_{p}\right)}{2}+U\left(h_{p}\right) \frac{\left(h_{p}+h_{f}-z\right)}{h_{f}} .
$$

Integration of these velocity profiles gives the granular and fluid flow rates,

$$
q_{p}=\frac{\Delta \rho g h_{f}^{3}}{\eta}\left[\frac{\phi}{6} \frac{\eta}{\eta_{e}}\left(\frac{h_{m}}{h_{f}}\right)^{3}\left(\frac{\partial p^{f} / \partial x}{\Delta \rho g}+\mu \phi\right)\right]
$$

and

$$
q_{f}=\frac{\Delta \rho g h_{f}^{3}}{\eta}\left\{-\frac{1}{12} \frac{\partial p^{f} / \partial x}{\Delta \rho g}+\frac{\eta}{\eta_{e}}\left(\frac{\partial p^{f} / \partial x}{\Delta \rho g}+\mu \phi\right)\left[\frac{1}{4}\left(\frac{h_{m}}{h_{f}}\right)^{2}+\frac{1-\phi}{6}\left(\frac{h_{m}}{h_{f}}\right)^{3}\right]\right\}
$$


respectively. The relevant scaling for the flow rate is thus $\Delta \rho g h_{f}^{3} / \eta$. It clearly does not involve the particle diameter as expected from the present continuum modelling. This calculation also shows that the control parameter is the dimensionless fluid flow rate.

For the more complex granular and suspension rheologies, equation (3.4) which is now nonlinear through the constitutive laws for $\mu\left(I_{v}\right), \phi\left(I_{v}\right)$, and $\eta_{e}(\phi)$ can be solved numerically using MATLAB. It is important to mention that the only assumption that we keep is that the Darcy drag term is dominant in the Brinkman equation (3.1) and that the problem is therefore reduced to simply solving the mixture equation (3.2). This important simplification is supported by the experimental evidence that there is very little slip between the two phases (see $\S 2$ ). The full three dimensional implementation of the original two-phase equations has been undertaken in an in-house finite element research code, in which the frictional granular rheology is handled using a regularisation technique (Chauchat \& Médale 2010). In this latter numerical model, the fluid-bed interface is fixed and no-slip boundary conditions are imposed on the walls for the mixture.

\section{Comparison}

Figures $5(\mathrm{a}, \mathrm{b})$ show the experimental data (taken in the middle plane of the rectangular channel) for $q_{v}$ and $h_{m}$, respectively, versus fluid height $h_{f}$. Clearly, $q_{v}$ and $h_{m}$ present a decrease with increasing $h_{f}$ which strongly depends on flow-rate as well as on particle and fluid combination. A good collapse is obtained in figure $5(\mathrm{c}, \mathrm{d})$ when using the scalings coming from the continuum approach described in $\S 3$. This suggests that the dimensionless fluid flow-rate $q_{f} /\left(\Delta \rho g h_{f}^{3} / \eta\right)$ is a good control parameter and that $h_{f}$ and $\eta / \Delta \rho g h_{f}$ can be taken as the length scale and time scale, respectively. These scalings are used in the following. An alternative scaling commonly found in the literature is to use the particle diameter $d$ as the length scale and the Shields number as the control parameter, see figures $5(\mathrm{e}, \mathrm{f})$. The collapse of the data presents slightly larger scatters but this can still be seen as appropriate.

We now turn to a quantitative comparison with the two-phase modelling using the (i) Coulomb and (ii) granular rheologies in figure 6 . We have also selected typical velocity profiles corresponding to different runs for the two combinations A and B (see table 2) at three different dimensionless flow rates in figure 7 . The good collapse of the different profiles for each dimensionless flow rate shows again that the scalings given by the continuum modelling of $\S 3$ are relevant.

The Coulomb rheology using a realistic $\mu_{s}=0.32$ (Boyer et al. 2011) and an Einstein effective viscosity $\eta_{e} / \eta=2.4$ yields sensible $q_{v}$ but fails in predicting $h_{m}$ (see the magenta dashed-dotted lines in figure 6). This discrepancy can be evidenced on the velocity profiles of figure 7 . The predicted flow inside the mobile layer gives larger velocity at the fluid-bed interface and smaller thickness of the mobile layer [see the magenta dashed-dotted lines in figure 7 (b), (d), and (f)]. These two effects compensate each other to give reasonable predictions for $q_{v}$ but both the velocity profile and the thickness of the mobile layer $h_{m}$ are not correctly described.

Instead of using the above commonly used values for $\mu_{s}$ and $\eta_{e} / \eta$, the method of least squares can be used to fit the data for $q_{v}, h_{m}$, as well as the interface velocity and leads to a better agreement for a smaller $\mu_{s}=0.24$ and a larger $\eta_{e} / \eta=14$ (see the green dashed lines in figure 6). Good agreement is also found for the velocity profiles at small flow rates [see the green dashed lines in figure $7(\mathrm{a}),(\mathrm{b}),(\mathrm{c}),(\mathrm{d})]$. At large flow rates, this model gives good prediction in the fluid region but leads to large overestimation in the mobile granular layer [see the green dashed lines in figure $7(\mathrm{e}),(\mathrm{f})]$. This overestimation 
(a)

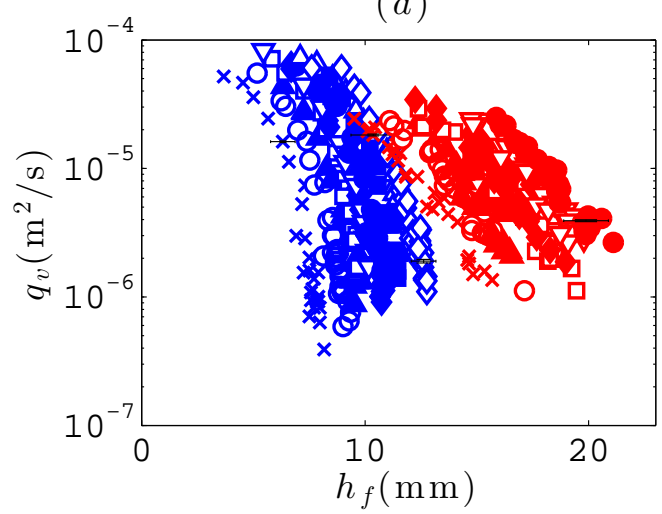

$(c)$

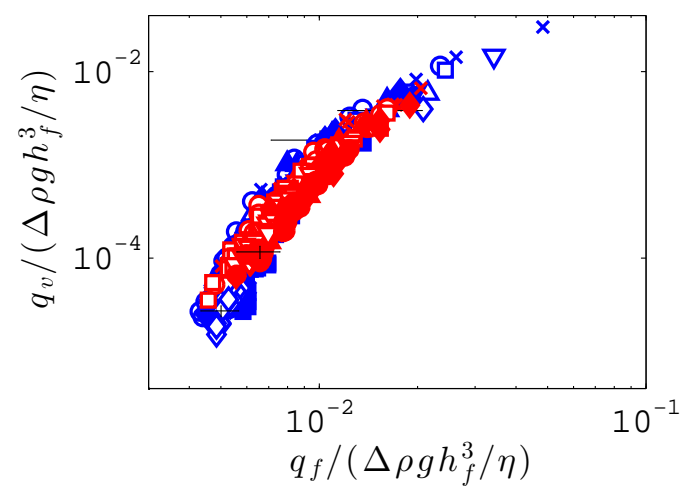

$(e)$

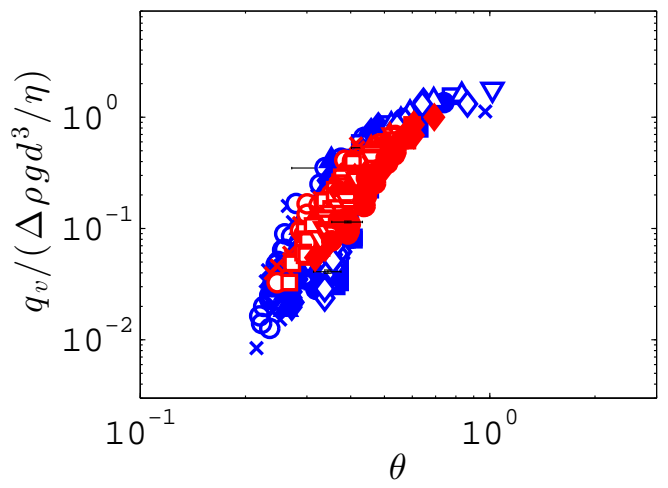

(b)

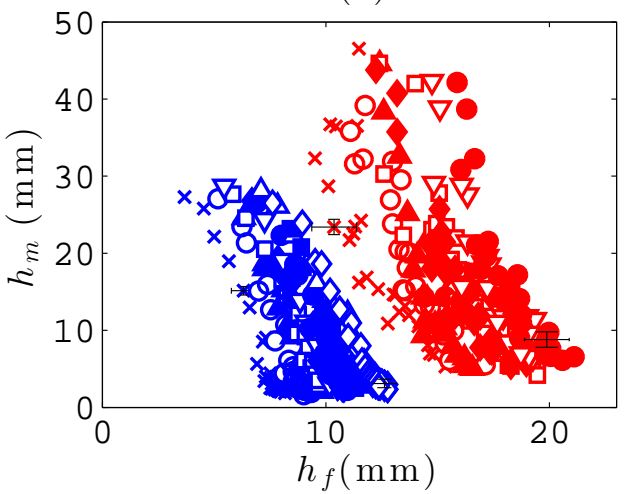

$(d)$
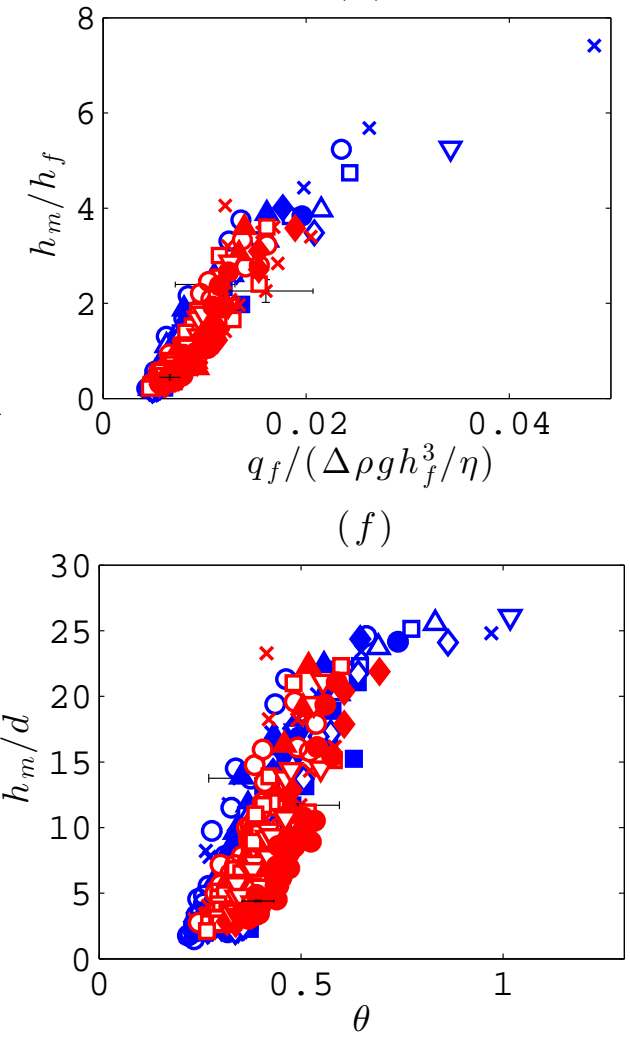

FiguRE 5. (a) Experimental particle-velocity flux $q_{v}$ and (b) thickness of the mobile layer $h_{m}$ versus fluid height $h_{f}$ for the different runs listed in table 2 for the different combinations A (blue symbols online) and B (red symbols online). (c) Particle-velocity flux $q_{v}$ made dimensionless by $\Delta \rho g h_{f}^{3} / \eta$ and (d) thickness of the mobile layer made dimensionless by $h_{f}$ versus fluid flow-rate $q_{f}$ made dimensionless by $\Delta \rho g h_{f}^{3} / \eta$ (e) Particle-velocity flux $q_{v}$ made dimensionless by $\Delta \rho g d^{3} / \eta$ and (f) thickness of the mobile layer $h_{m}$ made dimensionless by $d$ versus Shields number $\theta$. 
(a)

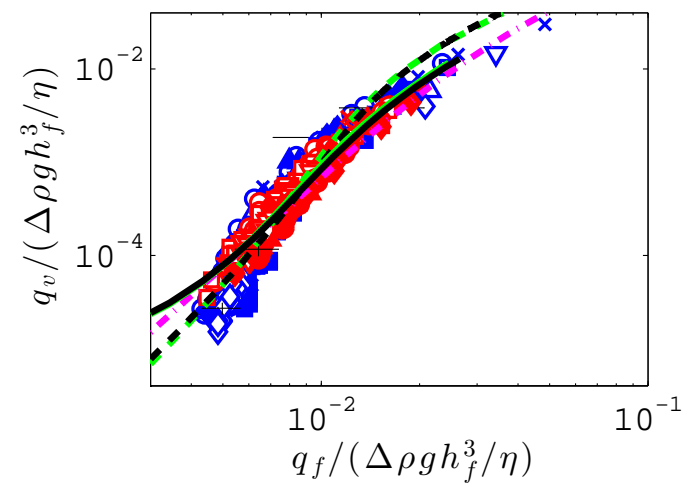

(b)

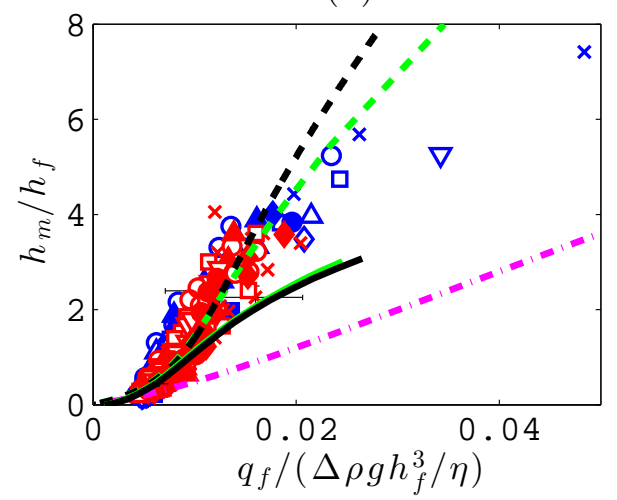

Figure 6. Dimensionless (a) particle-velocity flux $q_{v} /\left(\Delta \rho g h_{f}^{3} / \eta\right)$ and (b) thickness of the mobile layer $h_{m} / h_{f}$ versus dimensionless flow-rate $q_{f} /\left(\Delta \rho g h_{f}^{3} / \eta\right)$ compared to the two-phase model using (i) the Coulomb rheology with $\mu_{s}=0.32$ and $\eta_{e} / \eta=2.4$ (magenta dashed-dotted line) and with $\mu_{s}=0.24$ and $\eta_{e} / \eta=14$ (2D: green dashed line and 3D: green solid line for $h_{f}=8$ $\mathrm{mm}$ ) and (ii) the granular rheology with $\mu_{1}=0.24, \mu_{2}=0.39, I_{0}=0.01$, and $\eta_{e} / \eta_{f}=6.6$ (2D: black dashed line and 3D: black solid line for $h_{f}=8 \mathrm{~mm}$ ). The symbols corresponds to the different runs for the two different combinations listed in table 2.

can also be seen in figure 6 above a dimensionless flow-rate of $\approx 10^{-2}$ for which the bed-load thickness is of the order of the channel size.

Obviously, three-dimensional effects must be taken into account as seen by the better agreement with the three-dimensional numerical simulations for $q_{v}$ at large flow rate [see the green solid lines in figure 6(a)]. Note that there is some discrepancy at small flow rates due to numerical problems linked to the regularisation technique. The thickness of the mobile layer $h_{m}$ is also underestimated at large flow rate [see the green solid lines in figure 6(b)]. Note that the poor quantitative agreement obtained for $h_{m}$ is also due to the great difficulty in determining accurately $\dot{\gamma}^{p}=0$ on the velocity profiles both in the experiments and in the computations. Similar behaviour can be seen on the velocity profiles of figure 7. At relatively small flow rate [see figure 7(a), (b), (c) (d)] both twoand three-dimensional predictions are superimposed and are in good agreement with the experimental data. At larger flow rate [see figure 7(e), (f)] the two-dimensional model provides good predictions in the fluid region but yields an overestimation in the mobile granular layer whereas the three dimensional model gives a good prediction inside the mobile layer and a slight overestimation in the pure fluid region.

Comparison with the more sophisticated granular rheology having a shear-dependent friction coefficient $\mu\left(I_{v}\right)$ is also given in figures 6 and 7 (2D: black dashed line and 3D: black solid line for $h_{f}=8 \mathrm{~mm}$ ). A best fit using the method of least squares provides good agreement with both experimental $q_{v}$ and $h_{m}$ for $\mu_{1}=0.24, \mu_{2}=0.39$, $I_{0}=0.01$, and $\eta_{e} / \eta_{f}=6.6$. Note that the fitting gives the same friction coefficient of 0.24 for the Coulomb and granular rheologies but different effective viscosities. Using the fitted Coulomb and granular rheologies lead to similar predictions for the two and three-dimensional cases.

Three dimensional effects have been examined more thoroughly in runs 5 and 15 detailed in table 2 for which the rectangular pipe is scanned to obtain a three-dimensional investigation of the flow field. Note that the two runs correspond to two different combinations of particles and fluid. Figure 8 shows $q_{v}$ and $h_{m}$ versus the two-dimensional flow rate $q_{f}$ using the geometrical coefficient $k$ which provides the switch from three 
$(a)$

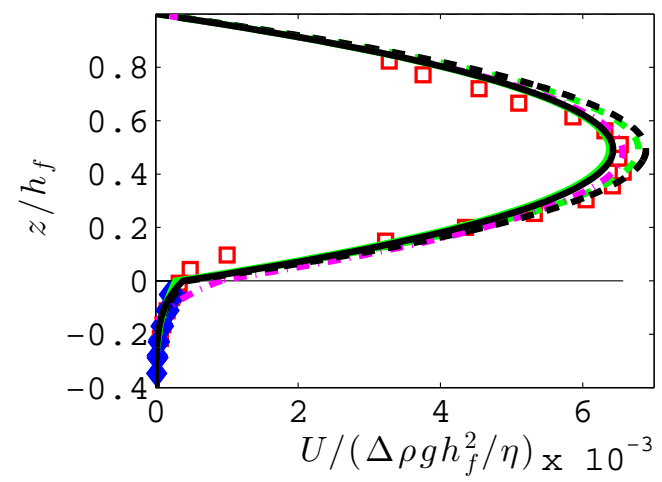

(c)

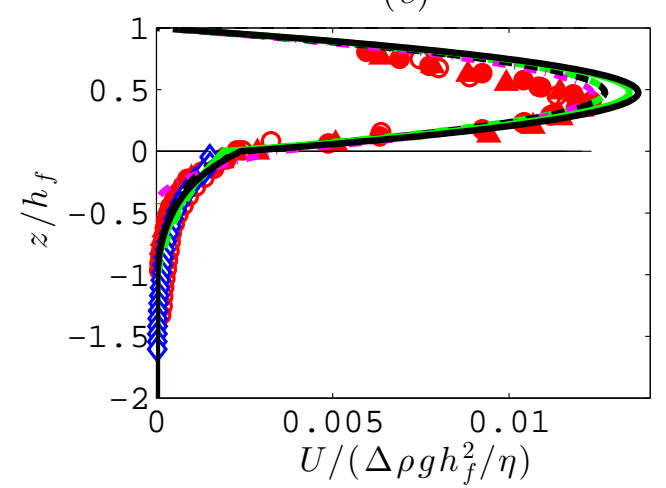

$(e)$

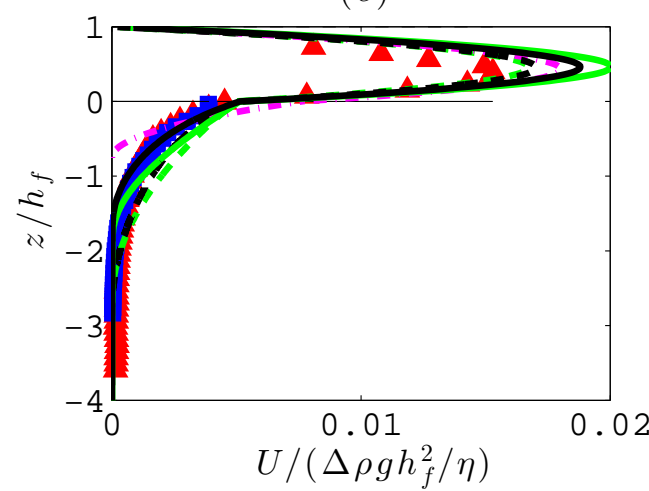

(b)

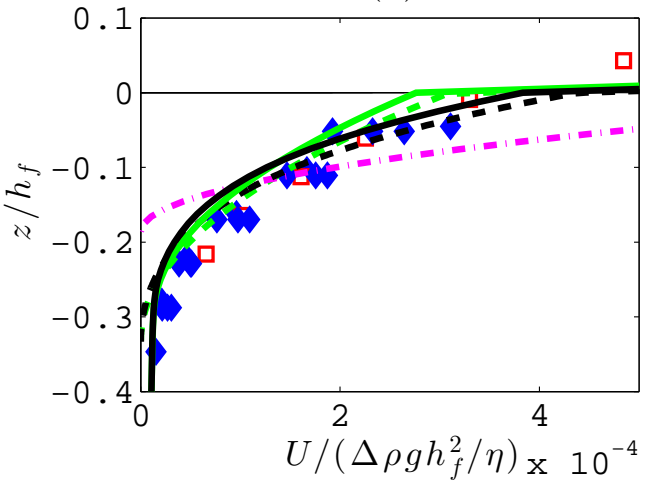

$(d)$

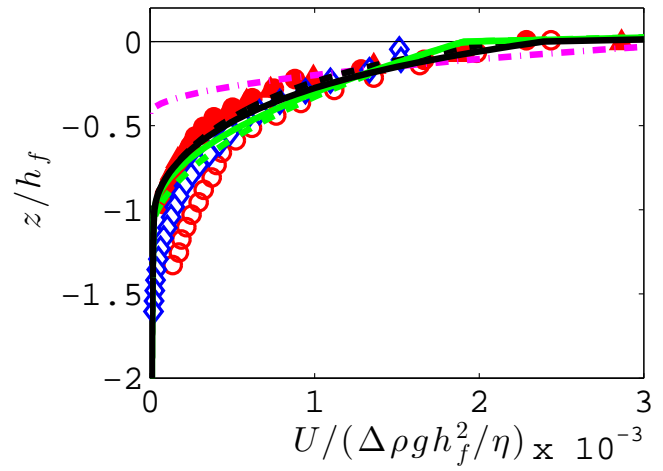

$(f)$

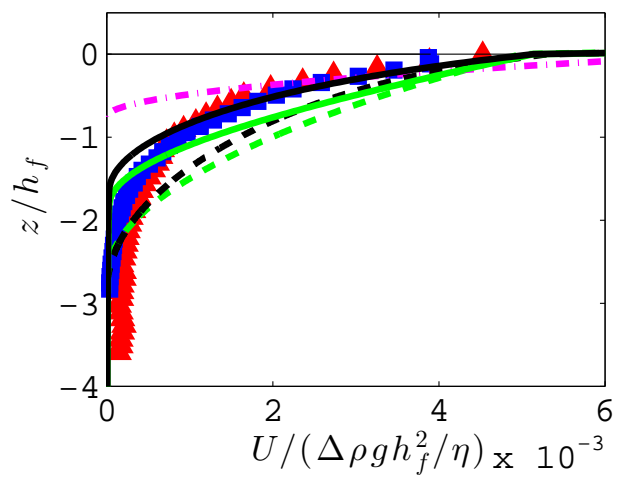

Figure 7. Comparison of the dimensionless experimental (symbols corresponding to the runs in table 2) and theoretical (same line representations as in figure 7 ) velocity profiles for 3 dimensionless flow-rate $q_{f} /\left(\Delta \rho g h_{f}^{3} / \eta\right)=4.610^{-3}, 9.110^{-3}, 13.910^{-3}$ from top to bottom. Graphs (b), (d), and (f) are blow-ups of graphs (a), (c), and (e), respectively.

to two-dimensional fluid flow-rate and depends on the location across the channel [see equation (2.1)]. Same good collapse and good agreements with the predictions of the three-dimensional numerical simulations taken in the middle plane are found except for the data close to the channel side where the granular motion is faster than predicted. This is a signature of a three-dimensional flow. 
(a)

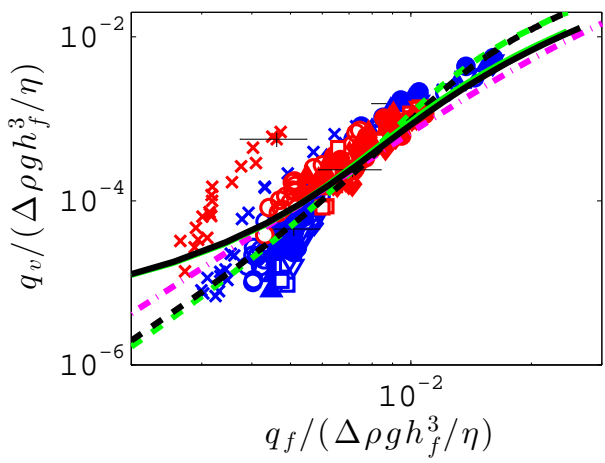

(b)

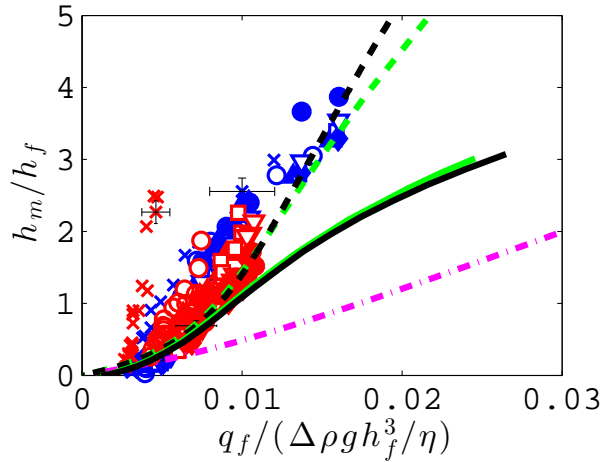

Figure 8. Same as figure 6 but for the scanned data across the channel: runs 5 (blue) and 15 (red) (symbols corresponding to the lateral position in table 2).

(a)

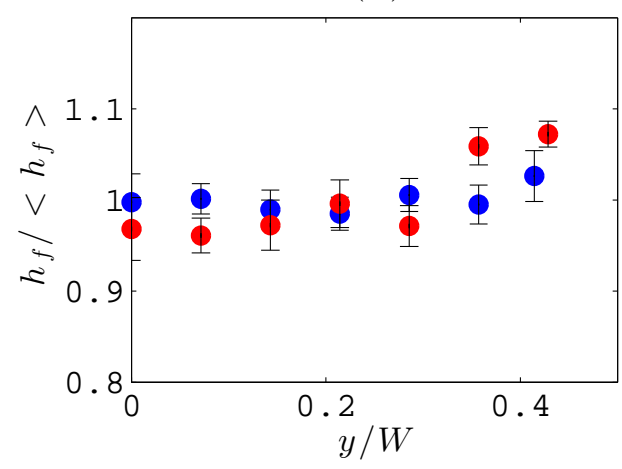

$(c)$

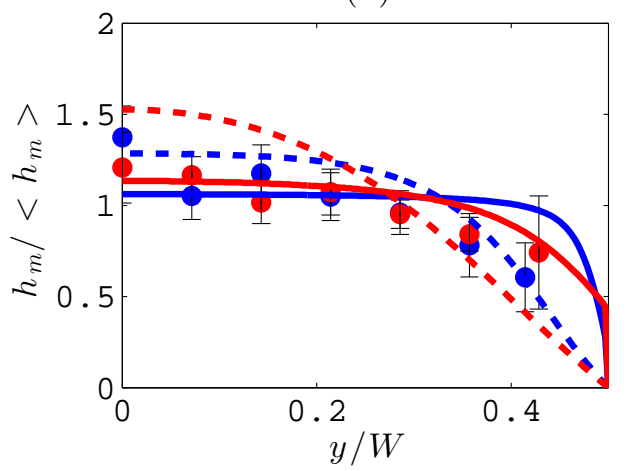

(b)

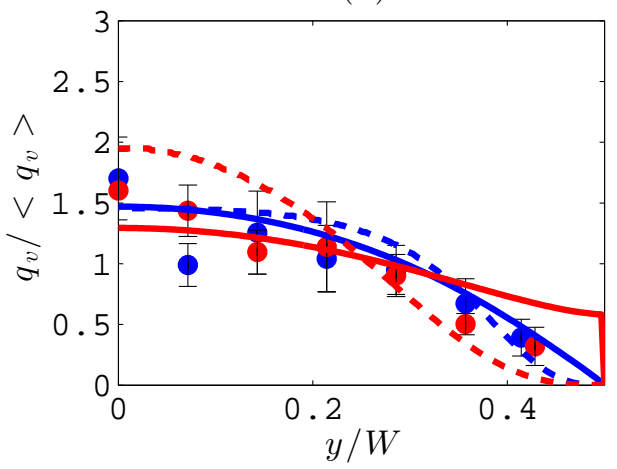

$(d)$

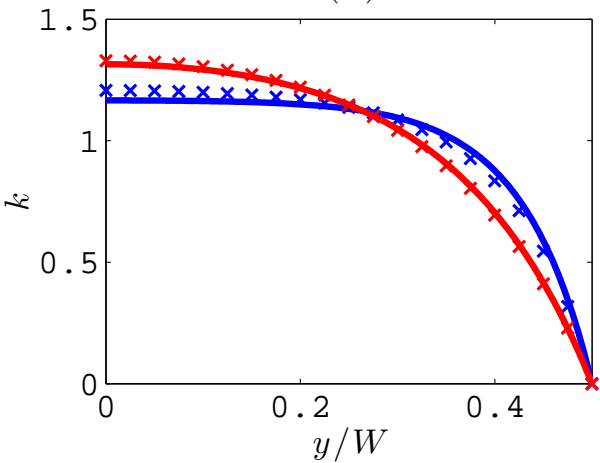

FIGURE 9. Variation across the channel of the averaged scanned measurements for runs 5 (blue, $h_{f} \subset[8-10] \mathrm{mm}$ ) and 15 (red, $h_{f} \subset[15-18] \mathrm{mm}$ ) detailed in table 2: (a) averaged fluid thickness normalised by the mean fluid thickness across the channel, (b) averaged particle-velocity flux $q_{v}$ normalised by the mean particle-velocity flux, (c) averaged thickness of the mobile layer $h_{m}$ normalised by the mean thickness of the mobile layer, and (d) geometrical coefficient $k$ obtained in the 3D numerical simulations using the granular rheology corresponding to run 5 conditions $\left(\times, h_{f}=8.1 \mathrm{~mm}\right)$ and to run 15 conditions $\left(\times, h_{f}=16.3 \mathrm{~mm}\right)$ and using equation (2.1) for $h_{f}=8.1 \mathrm{~mm}$ (blue solid line) and $h_{f}=16.3 \mathrm{~mm}$ (red solid line). Comparison with the two-phase model using the granular rheology with $\mu_{1}=0.24, \mu_{2}=0.39, I_{0}=0.01$, and $\eta_{e} / \eta_{f}=6.6$ [2D: dashed line and 3D: solid line for $h_{f}=8.1 \mathrm{~mm}$ (blue) and $h_{f}=16.3 \mathrm{~mm}$ (red)] is provided in graphs (b) and (c). 
$(a)$

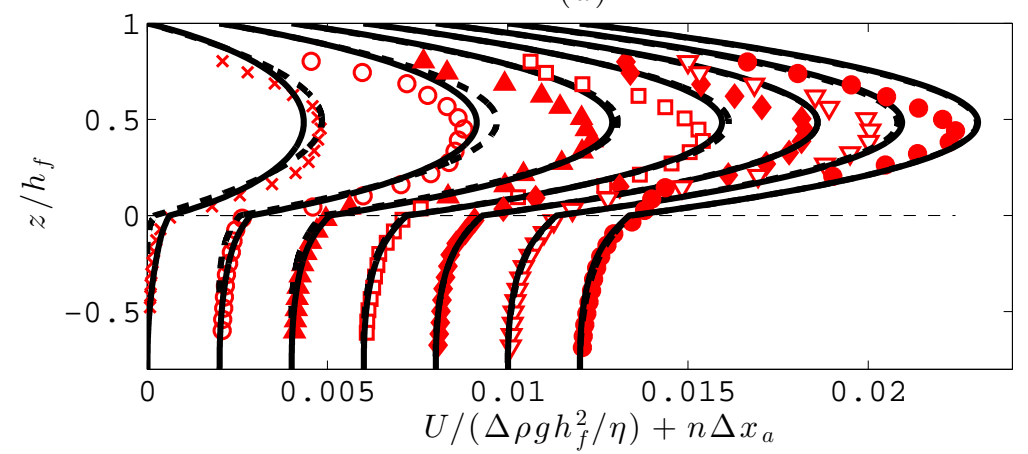

$(b)$

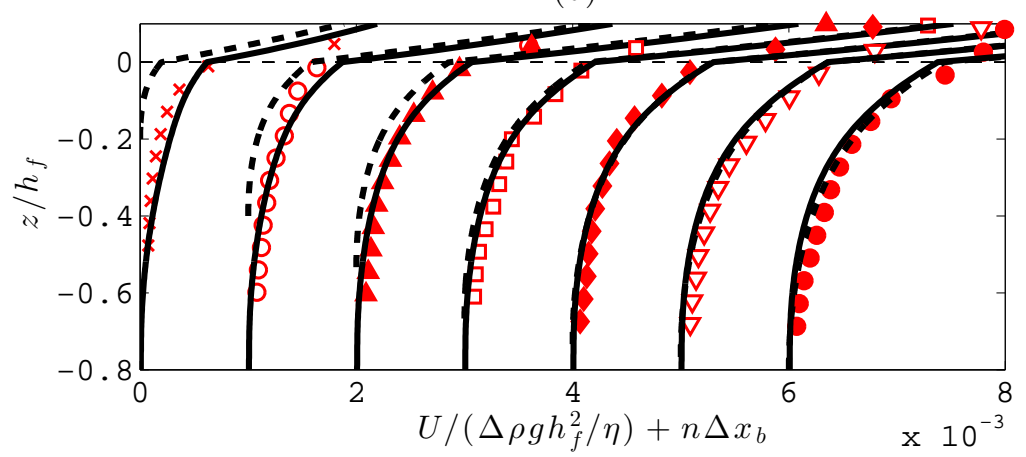

(c)

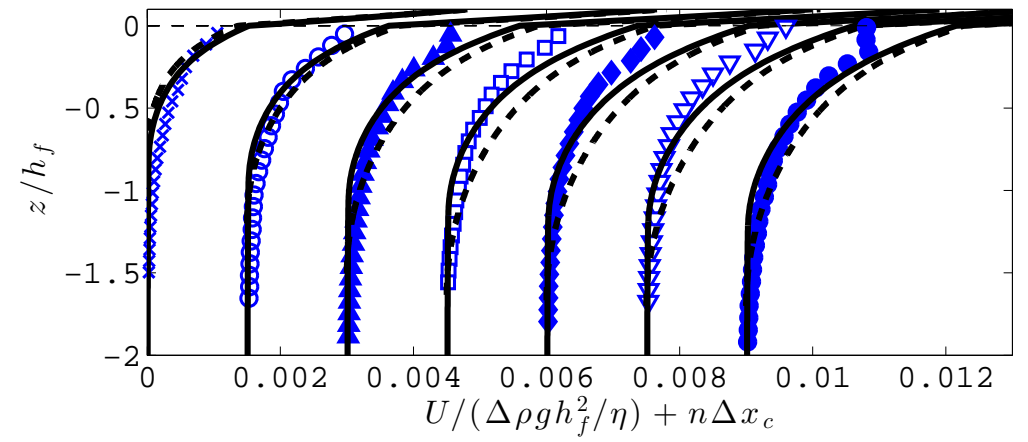

Figure 10. Comparison of the dimensionless velocity profiles between experiments corresponding to the different locations of (a, b) run 15 for $h_{f}=16.7 \mathrm{~mm}$ and (c) run 5 for $h_{f}=8.1 \mathrm{~mm}$ (symbols in table 2) and theoretical two-phase model using the granular rheology (2D: black dashed line and 3D: black solid line). The plots are shifted for clarity $\left(\Delta x_{a}=0.002, \Delta x_{b}=0.001\right.$, $\left.\Delta x_{c}=0.0015\right)$. Graphs (b) is a blow-up of graph (a).

Figure 9 presents the variation across the channel of $h_{f}, q_{v}, h_{m}$ and $k$. The fluid sediment interface is approximately flat across the pipe except close to the channel sides for which it becomes slightly lower on the order of a particle size. The three-dimensional predictions provide a better agreement for the variation of $q_{v}$ and $h_{m}$ than the twodimensional calculations (both using the granular rheology). The geometrical coefficients inferred from the predictions of the numerical simulations using the granular rheology and from equation (2.1) are in excellent agreement. This indicates that, in the present flow regime, the perturbation induced by the granular motion on the flow is weak and 
$(a)$

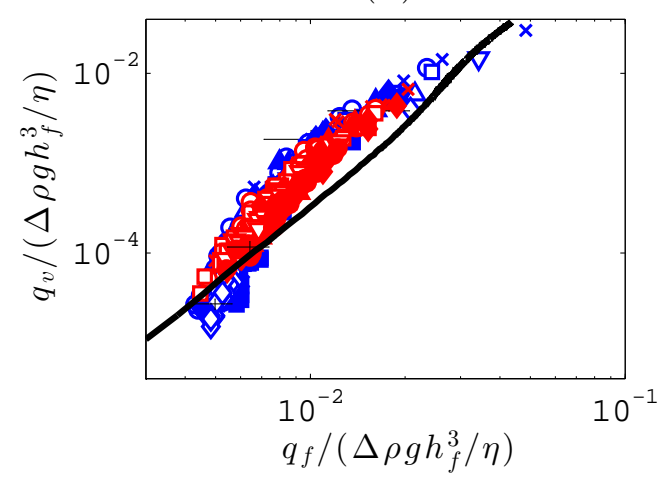

(b)

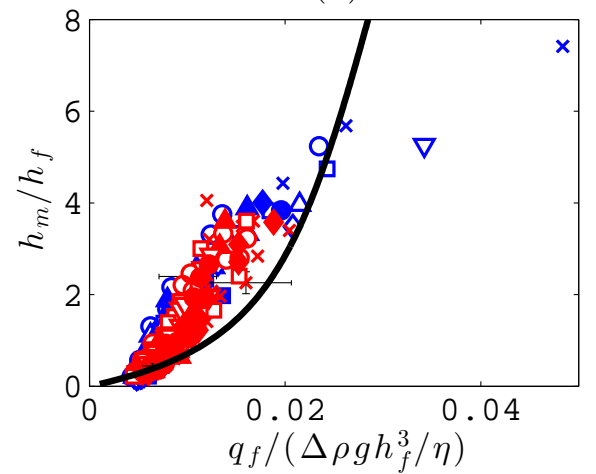

$(c)$

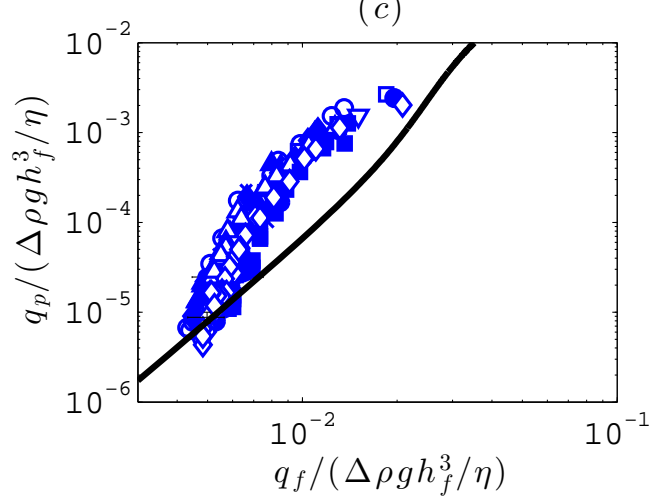

Figure 11. (a) and (b) Same as figure 6 and (c) particle flux $q_{p} /\left(\Delta \rho g h_{f}^{3} / \eta\right)$ with comparison to the two-phase model using the suspension rheology of Boyer et al. (2011) (2D: solid line).

thus that the use of this coefficient is valid. Note that the flow corresponding to run 5 $\left(h_{f}=8.1 \mathrm{~mm}\right)$ presents a much wider flat range of $k$ than that corresponding to run $15\left(h_{f}=16.3 \mathrm{~mm}\right)$. Figure 10 show that the fluid velocity profiles are well described by both the three- and two-dimensional calculations using the granular rheology. Inside the mobile sediment the three-dimensional predictions provide again a better agreement for the velocity profile, specially close to the side walls where the experiments show that the granular motion is faster than the $2 \mathrm{D}$ predictions. The discrepancy between the data and the three-dimensional calculation may be due to the no-slip boundary condition used in the three-dimensional simulations.

Finally, we provide comparison with the two-phase modelling using the more complex constitutive law recently proposed by Boyer et al. (2011) in figures 11 and 12. This new rheology for which none of the parameters has been adjusted gives the good order of magnitude. However, the predicted velocity profile is much stiffer and this yields an underestimation of both $q_{p}, q_{v}$, and $h_{m}$. In figure 12 , we have also given the variation of $\phi$ along $z$. Good agreement is found between the model and the experiments. The volume fraction $\phi$ is nearly constant along $z$ except on typically two particle diameters at the top where it rapidly goes to zero. This gives grounds for having a constant particle volume fraction in the previous Coulomb and granular models. 
(a)

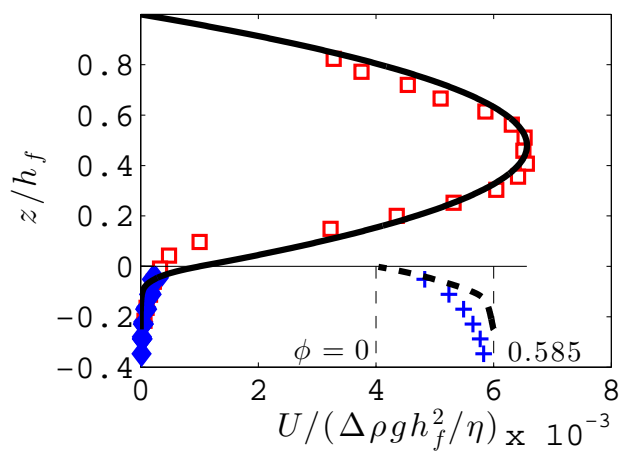

$(c)$

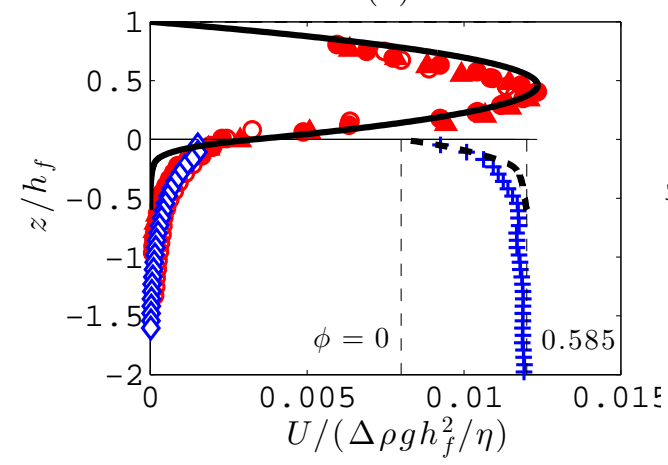

$(e)$

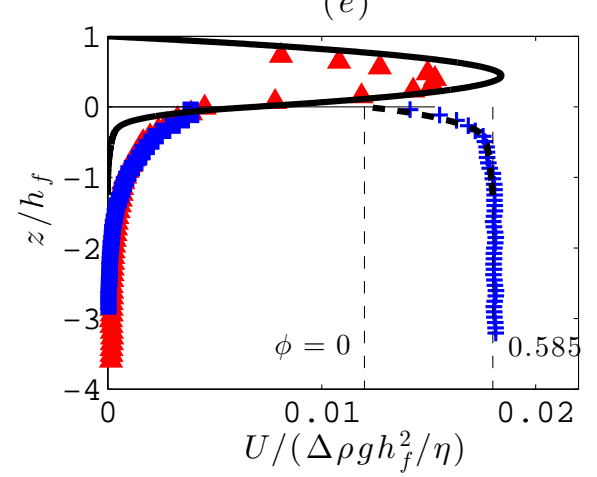

(b)

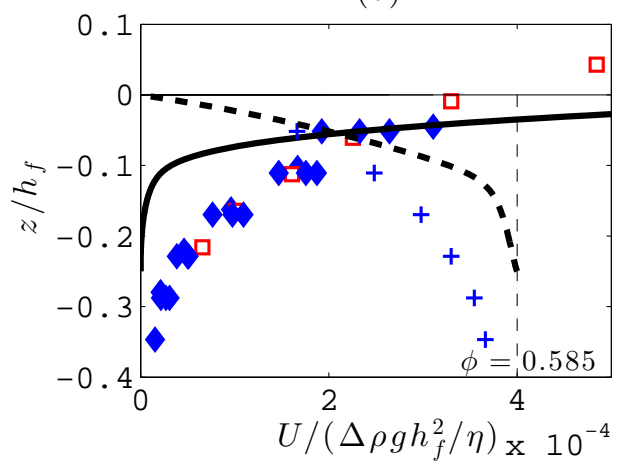

$(d)$

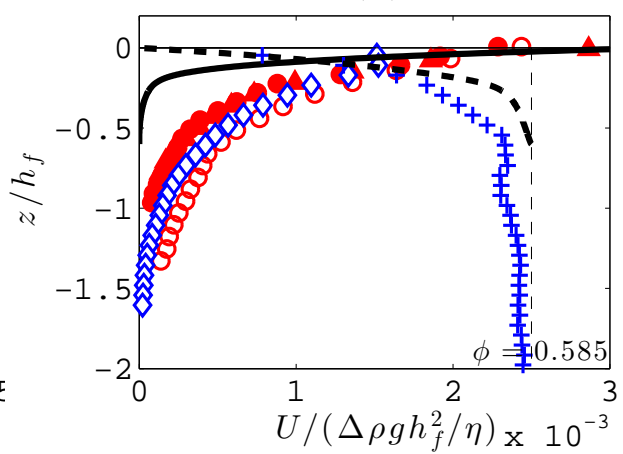

$(f)$

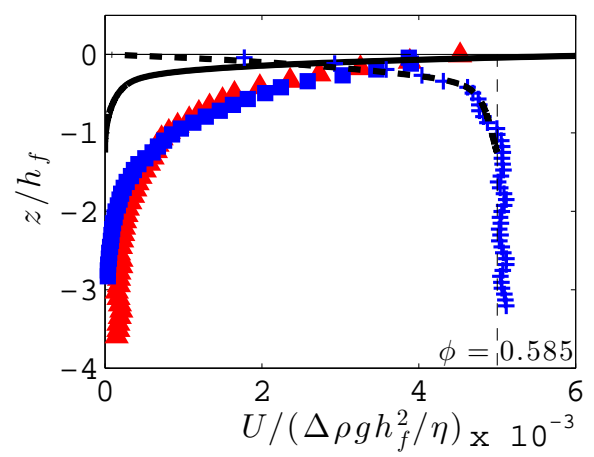

Figure 12. Same as figure 7 but with comparison to the two-phase model using the suspension rheology of Boyer et al. (2011) (2D: solid line). The variation of $\phi$ along $z$ is also represented (blue + : experiments and black dashed line: 2D predictions).

\section{Discussion and conclusion}

We have investigated the mobile layer of a granular bed composed of spherical particles in a laminar rectangular-channel flow. We have measured both particle and fluid velocities using particle image velocimetry for different index-matched combinations of particles and fluid and for a wide range of fluid flow-rate above incipient motion. An analysis of the velocity and concentration profiles inside the mobile granular layer shows that (i) there is no significant velocity slip between the particles and the fluid and (ii) the particle volume fraction is approximately constant except at the bed interface where it vanishes on typically two particle diameters. An investigation of the appropriate scalings shows 
that adopting the fluid height as the length scale and a viscous time scale as suggested by a continuum modelling gives a good collapse of the data for the particle flux, thickness of the mobile layer, and velocity profiles.

The measurements are then compared to a two-phase continuum approach having a frictional rheology for the particle phase and a Newtonian rheology for the fluid phase originally proposed by Ouriemi et al. (2009). The simplest constitutive model using a realistic Coulomb friction coefficient of 0.32 and an Einstein viscosity and assuming uniform particle concentration inside the mobile layer yields realistic particle flux but fails in predicting the bed-load thickness as well as the velocity profiles. Good agreement is obtained by fitting the two parameters of this rheology, i.e. the friction coefficient and the effective viscosity. The obtained value for the friction coefficient is 0.24 and is smaller than that found in rheological measurements (Boyer et al. 2011) while the value for the effective viscosity is 14 and is much larger than that used in the porous media literature (see e.g. Goharzadeh et al. 2005). This large viscosity is able to describe the slope of the velocity profile at the interface but is not realistic. This inconsistency can be overcome by using a shear-dependent friction rheology (having the same static friction coefficient of 0.24 ) which provides excellent agreement with a lower effective viscosity of 6.6. In addition, this rheology implemented in a full three-dimensional simulation is able to describe three dimensional effects observed at larger flow rates as well as the flow variation across the channel. The two friction coefficients $\mu_{1}$ and $\mu_{2}$ in the $\mu(I)$ rheology are slightly lower than those expected. This may be due to additional phenomena such as dilatancy which, even small, could act as reducing friction in the present experimental configuration. The more complex rheological model proposed by Boyer et al. (2011) having no adjustable parameters gives the proper trend and good order of magnitude but fails in describing the velocity profile. This third model comes from rheological measurements of dense suspensions of neutrally-buoyant spheres using pressure-imposed rheometry and may not be directly applicable to buoyant suspensions with a strong gradient of concentration at the particle-fluid interface. Again dilatancy effect should also be included in this model.

To conclude, a two-phase continuum model having a frictional rheology is able to predict quantitatively the flow inside the mobile layer of a sheared granular media. Three rheological constitutive laws having increasing degree of sophistication have been tested and discussed. Clearly among these three rheological models, the granular model is the most successful but the simplest analytical Coulomb model still gives a sensible prediction for the particle flux. It would be good to have in the future a robust rheological model employed to predict behaviour to a wide class of dense particulate flows. The present experimental data can be used as a benchmark for rheological models in continuum approach but also for particle-interaction model in discrete-particle simulations.

\section{Acknowledgements}

Funding from Agence Nationale de la Recherche (Project Dunes ANR-07-3 18-3892) is gratefully acknowledged.

\section{REFERENCES}

BAgnold, R. A. 1956 The flow of cohesionless grains in fluids. Phil. Trans. R. Soc. Lond. A 249, 235-297.

BAGNOLD, R. A. 1954 Experiments on a gravity-free dispersion of large solid spheres in a Newtonian fluid under shear. Proceedings of the Royal Society of London. Series a, Mathematical and Physical Sciences, 225, 49-63. 
Boyer, F., Guazzelli, É. \& Pouliquen, O. 2011 Unifying Suspension and Granular Rheology. Phys. Rev. Lett. 188301.

Brinkman, H. C. 1947 A calculation of the viscous force exerted by a flowing fluid on a dense swarm of particles. Appl. Sci. Res. A1, 27-34.

Cassar, C., Nicolas \& Pouliquen, O. 2005 Submarine granular flows down inclined planes. Phys. Fluids 17, 103301.

Chauchat, J., \& MÉdale, M. 2010 A three-dimensional numerical model for incompressible two-phase flow of a granular bed submitted to a laminar shearing flow. Comput. Methods Appl. Mech. Engrg., 199, 439-449.

Charru, F., Mouilleron-Arnould, H. \& Eiff, O. 2004 Erosion and deposition of particles on a bed sheared by a viscous flow. J. Fluid Mech. 519, 55-80.

Derksen, J. 2011 Simulations of granular bed erosion due to laminar shear flow near the critical Shields number. Phys. Fluids 23113303.

Dijksman, J. A., Rietz, F., Lõrincz, K. A.,VAn Hecke, M. \& Losert, W. 2012 Invited Article: Refractive index matched scanning of dense granular materials. Rev. Sci. Instrum. 83, 011301.

Forterre, Y. \& Pouliquen, O. 2008 Flows of dense granular media. Annu. Rev. Fluid Mech. 40, $1-24$.

Goharzadeh, A., Khalili, A. \& Jørgensen, B. B. 2005 Transition layer thickness at a fluid-porous interface. Phys. Fluids 17, 057102.

JACKSON, R. 1997 Locally averaged equations of motion for a mixture of identical spherical particles and a Newtonian fluid. Chemical Engineering Science 52, 2457-2469.

JACKSON, R. 2000 The dynamics of fluidized particles, Cambridge University Press.

Lajeunesse, E., Malverti, L., Lancien, P., Armstrong, L., Metivier, F., Coleman, S., Smith, C. E., Davies, T., Cantelli, A. \& Parker, G. 2010 Fluvial and submarine morphodynamics of laminar and near-laminar flows: a synthesis. Sedimentology 57,1-26

Lobkovsky, A. E., Orpe, A. V., Molloy, R., Kudrolli, A. \& Rothman, D. H. 2008 Erosion of a granular bed driven by laminar fluid flow. J. Fluid Mech. 605, 47-58.

Loiseleux, T., Gondret, P., Rabaud, M. \& Doppler, D. 2005 Onset of erosion and avalanches for an inclined granular bed sheared by a continuous laminar flow. Phys. Fluids $17,103304$.

Malverti, L., Lajeunesse, E. \& Métivier, F. 2008 Small is beautiful: Upscaling from microscale laminar to natural turbulent rivers. J. of Geophys. Research, 113 F04004.

Meunier, P. \& Leweke, T. 2003 Analysis and minimization of errors due to high gradients in Particle Image Velocimetry. Exp. Fluids, 35(5) 408-421.

Mouilleron, H., Charru, F. \& Eiff, O. 2009 Inside the moving layer of a sheared granular bed. J. Fluid Mech. 628, 229-239.

Nicolas, X., LuiJkx, J.-M. \& Platten, J.-K. 2000 Linear stability of mixed convection flows in horizontal rectangular channels of finite transversal extension heated from below, International Journal of Heat and Mass Transfer 43 589-610.

Ouriemi, M., Aussillous, P., Médale, M., Peysson, Y. \& Guazzelli, É. 2007 Determination of the critical Shields number for particle erosion in laminar flow. Phys. Fluids 19, 061706 .

Ouriemi, M., Aussillous, P. \& Guazzelli, É. 2009 Sediment dynamics. Part 1. Bed-load transport by laminar shearing flows. J. Fluid Mech. 636, 295-319.

Van der Hoef M. A., Beetstra, R. \& Kuipers J. A. M. 2005 Lattice-Boltzmann simulations of low-Reynolds-number flow past mono- and bidisperse arrays of spheres: results for the permeability and drag force. J. Fluid Mech., 528, 233-254.

Van RiJn, L. C. 1984. Sediment Transport, Part I: Bed Load Transport. Journal of Hydraulic Engineering, 110(10), 1431-1456. 